\title{
El rol de la inteligencia emocional y del conflicto trabajo- familia en la satisfacción laboral, el desempeño percibido y la intención de abandono de los docentes
}

\author{
Cristian A. Delgado-Bello1, Iván A. Veas-González², Marcelo R. Avalos-Tejeda ${ }^{3}$, Orlando F. Gahona-Flores ${ }^{4}$ \\ (1) Escuela de Ingeniería Comercial, Facultad de Economía y Negocios, Universidad Santo Tomás, Antofagasta, Chile. \\ (correo-e: cristiandelgadobe@santotomas.cl) \\ (2) Departamento de Administración, Facultad de Economía y Administración, Universidad Católica del Norte, \\ Antofagasta, Chile. (correo-e: iveas@ucn.cl) \\ (3) Escuela de Psicología, Facultad de Ciencias Sociales, Universidad Santo Tomás, Antofagasta, Chile. \\ (correo-e: marceloavaloste@santotomas.cl) \\ (4) Departamento de Ingeniería Comercial, Facultad de Ingeniería, Universidad de Antofagasta, Antofagasta, Chile. \\ (correo-e: orlando.gahona@uantof.cl)
}

Recibido Ago. 7, 2020; Aceptado Oct. 8, 2020; Versión final Dic. 3, 2020, Publicado Feb. 2021

\section{Resumen}

El objetivo de esta investigación es analizar la relación entre la inteligencia emocional (IE), el conflicto trabajo familia (CTF), la satisfacción laboral (SL), el desempeño (D) y la intención de dejar el trabajo (IDT), en el contexto de los docentes de educación primaria semi-particular de la región de Antofagasta (Chile). Fueron seleccionados 140 docentes ( $81 \%$ mujeres) a través de un muestreo no probabilístico por conveniencia. Este es un estudio de enfoque cuantitativo con medidas validadas en forma de cuestionarios de auto-respuesta. El análisis de los datos se realizó mediante el método de dos etapas en ecuaciones estructurales. Los resultados señalan que el modelo planteado tiene una significativa e importante capacidad de explicar el desempeño, la satisfacción laboral y la intención de dejar el trabajo de los docentes. Así mismo, es posible concluir que la inteligencia emocional juega un importante rol en la satisfacción laboral y el desempeño de los docentes.

\section{The role of emotional intelligence and work-family conflict in teacher job satisfaction, perceived performance, and turnover}

\begin{abstract}
This research study examines the relationships between emotional intelligence (EI), work-family conflict (WFC), job satisfaction (JS), performance (P), and turnover intention (TI) in teachers working at semi-private primary education schools from the city of Antofagasta (Chile). There were 140 teachers ( $81 \%$ females) selected through non-probability convenience sampling. The present study used a quantitative approach validated by self-response questionnaires. Data analyses were performed using the structural equations twostage method. The results indicate that the model proposed here can accurately explain teacher performance, job satisfaction, and turnover intention. It is concluded that emotional intelligence plays an important role in teacher job satisfaction and performance.
\end{abstract}




\section{INTRODUCCIÓN}

Excesivas demandas laborales pueden traer consecuencias no deseadas en el bienestar familiar de los trabajadores, y a su vez en el desempeño de estos, puesto que el rol laboral se puede expandir y sobreponer al rol familiar, originando frustración e insatisfacción en el desempeño de ambos roles. Este fenómeno es conocido como conflicto trabajo familia (CTF), y se define como una forma de conflicto inter-rol en el que las demandas de los ámbitos laboral y familiar son mutuamente incompatibles en algunos aspectos (Greenhaus y Beutell, 1985).

EI CTF, ha captado el interés de los investigadores del comportamiento organizacional, dando origen a una serie de trabajos que estudian y relacionan el CTF con el estrés, la satisfacción laboral (SL), el desempeño y más recientemente con la inteligencia emocional. Si bien, la mayor parte de la literatura se ha enfocado en el contexto de gerentes y directivos de empresas, se ha demostrado que el fenómeno puede variar en relaciones y efectos, según ocupación (Pousette y Hanse, 2002). Así el contexto educación, específicamente la ocupación docente, representa un caso interesante de estudio, no solo porque ha sido un contexto poco estudiado en la literatura, sino que además el trabajo de los docentes en Chile se suele extender más allá de su lugar de trabajo. Puesto que estos deben responder a altas exigencias laborales, asociadas con las demandas institucionales, ministeriales e inclusive de los apoderados. Si bien, la profesión docente presenta un escenario complejo, Gao et al. (2013) señalan que la inteligencia emocional (IE) puede ser un factor protector o moderador para los trabajadores sometidos a altas exigencias laborales. En este sentido, en la medida que los trabajadores, aumentan la regulación y comprensión de las emociones propias y de terceros, pueden mejorar su desempeño. De ahí, que el objetivo de esta investigación es analizar la relación entre la Inteligencia emocional (IE), el conflicto trabajo familia (CTF), la satisfacción laboral (SL), el desempeño percibido (D) y la intención de dejar el trabajo (IDT), en el contexto de los docentes de educación primaria (ver figura 1).

La IE ha sido conceptualizada desde diferentes enfoques. Desde el modelo de habilidad o capacidad, la IE se define como una capacidad mental para percibir, utilizar, comprender y gestionar las emociones propias y de los demás (Mayer y Salovey 1997). Por otra parte, en el modelo de rasgos cognitivos la IE se conceptualiza como una combinación de autopercepciones relacionadas con las emociones y disposiciones conductuales, asociada con la capacidad de reconocer y utilizar la información relacionada con las emociones (Bar-On; 1997; Shi y Wang, 2007), que se ubica en los niveles más bajos de las taxonomías de la personalidad (Petrides et al., 2007). En el contexto de la enseñanza y la investigación docente, es más frecuente el modelo de IE de rasgos (Yin et al, 2013). De acuerdo con Wong et al. (2010) la IE de los profesores comprende cuatro aspectos distintivos: valoración de la emoción en el yo; valoración o reconocimiento de emociones en otros; regulación de la emoción en el yo; y uso de la emoción para facilitar la actuación. En consideración de lo anterior, el presente estudio se centra en el modelo de rasgos de la IE, midiendo la IE de los profesores a través del cuestionario de autoinforme propuesto por Wong y Law, (2002).

Respecto de la relación entre la IE y la SL, ésta ha sido abordada por varios estudios y en diferentes áreas durante los últimos años, concluyendo que existe una correlación positiva entre estas variables (Wen et al., 2019). Los empleados con alta inteligencia emocional son más propensos a tener mejores niveles de satisfacción en el trabajo, ya que son expertos en la evaluación y la regulación de las emociones propias y ajenas (Sy et al., 2006), a diferencia de los empleados con menos inteligencia emocional, quienes son menos conscientes de sus emociones y poseen menos habilidades para hacer frente a estas en las situaciones diarias, agravando su nivel de estrés y disminuyendo su nivel de satisfacción laboral (Sy et al., 2006). En consecuencia, se desprende la siguiente hipótesis: H1: La inteligencia emocional tiene un efecto directo y positivo sobre la satisfacción laboral de los docentes de educación primaria.

Según Porter y Lawler (1991), el desempeño laboral, en general, se refiere a comportamientos que son relevantes para los objetivos organizacionales, y hay tres tipos de rendimiento. Una es la medida de las tasas de producción; la cantidad de ventas durante un período de tiempo determinado, la producción de un grupo de empleados que informan al gerente, etc. El segundo tipo de medida de desempeño involucra calificaciones de individuos por alguien que no sea la persona cuyo desempeño se está considerando. El tercer tipo de medidas de desempeño es la autoevaluación. Diversas investigaciones señalan que la IE tiene un efecto positivo en el desempeño laboral (Joseph et al., 2015). Los empleados con alta IE serían más propensos a ajustar sus propias emociones y manejar las emociones de los demás para crear interacciones más positivas, lo que podría conducir a un mejor desempeño (Wong y Law, 2002). Sy et al., (2006) encontraron que la inteligencia emocional es predictiva del desempeño laboral en una amplia variedad de contextos. En específico en el contexto educacional se puede señalar que los docentes con alta inteligencia emocional tienen más probabilidades de desempeñarse bien en su trabajo (Yoke y Panatik, 2015). Lo anterior lleva a plantear la siguiente hipótesis: H2: La inteligencia emocional tiene un efecto directo y positivo sobre el desempeño de los docentes de educación primaria. 


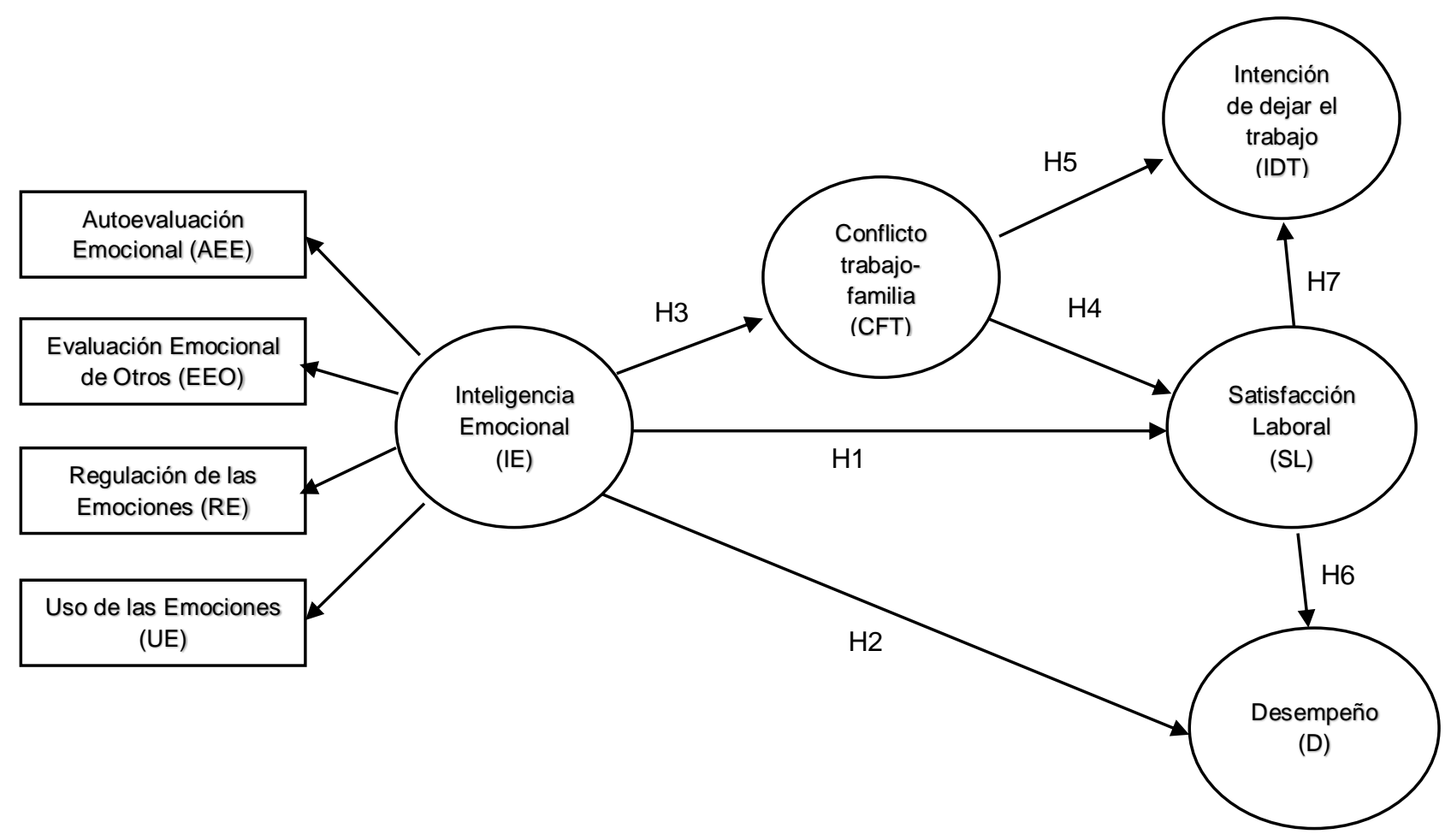

Fig. 1: Modelo propuesto

Respecto al conflicto trabajo-familia (CTF), este se define como una forma de conflicto inter-rol en el que las demandas de los ámbitos laboral y familiar son mutuamente incompatibles en algunos aspectos (Greenhaus y Beutell, 1985). El CTF resulta problemático tanto para trabajadores como empleadores ya que interfiere con el desempeño del trabajador en su lugar de trabajo, siendo común que el trabajador se vea aquejado de fatiga, sentimientos de incompetencia e insatisfacción laboral (Erdamar y Demirel, 2014). Sin embargo, Gao et al. (2013) mostraron que la inteligencia emocional reduce el estrés causado por el conflicto trabajo-familia. En otros trabajos también se muestra una relación negativa entre inteligencia emocional y conflicto trabajo-familia, como por ejemplo el Cheung y Tang, (2012) concluyen que la regulación emocional puede ayudar a los empleados a mantener estados afectivos de signo positivo, lo que contribuiría a la mejora del rendimiento en el trabajo y el funcionamiento familiar. Lo que nos lleva a plantear la siguiente hipótesis: H3: La inteligencia emocional tiene un efecto directo y negativo sobre el conflicto trabajo familia de los docentes de educación primaria.

Por otra parte, el CTF aumenta la intención de dejar el trabajo (IDT), tanto en forma directa, como indirectamente al disminuir la satisfacción con el trabajo ya que al disminuir ésta, aumenta la IDT (Erdamar y Demirel, 2016). En instituciones educativas este fenómeno se ha observado en docentes de diversos niveles educativos y culturas. Erdamar y Demirel (2014) observan en profesores de primaria y secundaria en Ankara (Turquía) que los conflictos más comúnmente reportados son que el trabajo se impone al tiempo que pasan en familia y los mantiene física y mentalmente fatigados. Gao et al. (2013) observan en profesores secundarios de China que el CTF se relaciona negativamente con la satisfacción laboral y que la IE modera esta relación. En una muestra más diversa, que abarcó profesores desde preescolar hasta secundaria, Erdamar y Demirel (2016) hallan que el CTF se relaciona negativamente con la satisfacción con el trabajo y la vida, siendo mayor el CTF en mujeres que en hombres y en profesores de primaria que en los demás niveles. En consecuencia, se desprenden la siguientes hipótesis: H4: El conflicto trabajo-familia tiene un efecto directo y positivo sobre la intención de dejar el trabajo de los docentes de educación primaria; y H5: El conflicto trabajo-familia tiene un efecto directo y negativo sobre la satisfacción laboral de los docentes de educación primaria.

Varios autores señalan que la relación entre la satisfacción laboral y el desempeño es muy clara y directa, siendo la satisfacción un importante predictor del desempeño (Patterson et al., 2004). Se ha encontrado una relación significativa y negativa entre la satisfacción laboral y aspectos negativos del comportamiento organizacional tales como el ausentismo y la rotación en el trabajo, de modo que quienes están más insatisfechos presentan mayores tasas de absentismo y rotación. Así, Patterson et al. (2004), señalan que la satisfacción laboral se ha relacionado más con el desempeño, cuando éste es medido a través de aspectos económicos más bien relacionados con la productividad. Por último, Bustamante et al. (2020) señala que es necesario gestionar las variables que determinan la satisfacción, ya que esta, tiene un efecto inmediato en el rendimiento o desempeño laboral. Lo 
anterior nos lleva a plantear la siguiente hipótesis: H6: La satisfacción laboral tiene un efecto directo y positivo sobre el desempeño de los docentes de educación primaria.

De acuerdo con Johnsrud y Rosser (2002), la satisfacción del profesorado con su trabajo académico, así como otras características personales, estructurales y del entorno organizacional, han sido consideradas relevantes en la explicación de la intención de los académicos de abandonar su trabajo; esta intención, además, es considerada un indicador aproximado del abandono factual de la misma. Los autores encontraron que el principal aspecto relacionado con este hecho fue la percepción de los académicos acerca de la vida laboral en función de las prioridades y recompensas profesionales, el apoyo de la administración y el tipo de relación con ésta, así como la calidad de los beneficios y servicios. En consecuencia, se desprende la siguiente hipótesis: H7: La satisfacción laboral tiene un efecto directo y negativo sobre la intención de dejar el trabajo de los docentes de educación primaria.

\section{METODOLOGÍA}

Para alcanzar el objetivo de investigación, es necesario emplear un método que permita medir y analizar los resultados de variables que no son directamente observables. Para esto, se diseñó una investigación cuantitativa de corte transversal, a través de un muestreo no probabilístico por conveniencia y análisis de datos basados en ecuaciones estructurales. El cuestionario fue estructurado, según los instrumentos de medición empleados en la literatura internacional y en los trabajos para Latinoamérica (ver Tabla 1). Replicando la escala de Likert de 5 puntos, donde 1 representa a la respuesta "Muy en Desacuerdo" y 5 "Muy de Acuerdo". Se realizó una prueba piloto con 30 sujetos para comprobar la validez del instrumento. El cuestionario fue enviado a 300 docentes previamente contactados pertenecientes a 2 establecimientos particulares de nivel primario, de la ciudad de Antofagasta. Se recibieron 140 respuestas entre los meses junio - agosto del año 2018. En donde la edad promedio de la muestra fue de 35 años, con un $65 \%$ de los sujetos entre 23 y 35 años, de la muestra total el $81 \%$ fueron mujeres, además el $44 \%$ son solteros y el $50 \%$ son casados o convivientes.

En consideración que las variables no son directamente observables, el análisis y evaluación del modelo se realizó a través de la técnica de partial least square (PLS). PLS-SEM permite a los investigadores evaluar tanto relaciones causales entre indicadores/ítems, así como también relaciones causales de constructos latentes (Gudergan et al., 2008). Para evaluar las mediciones y el modelo estructural se utilizaron los procedimientos sugeridos en la literatura previa (Fornell y Larcker, 1981). Se incluyó la medida de IE como una variable de segundo orden, reflectiva-reflectiva de acuerdo con Yin et al. (2013) recurriendo a la metodología de dos pasos de Wright et al. (2012). Los datos fueron analizados utilizando el software SmartPLS 3.0. PLS resuelve cuestiones relevantes que surgen al proponer ecuaciones estructurales: soluciones inadmisibles, indeterminación de factores y la no necesidad de una distribución normal de datos (Ringle et al., 2015).

Tabla 1: Origen y definición de las escalas de medición.

\begin{tabular}{|c|c|c|c|}
\hline Variable & Tipo de Variable & $\begin{array}{l}\text { Número de } \\
\text { Indicadores }\end{array}$ & Referencias \\
\hline Inteligencia Emocional & $\begin{array}{l}\text { Variable de } 2^{\circ} \text { Orden, } \\
\text { reflectiva }\end{array}$ & 16 & Wong y Law, (2002) \\
\hline Satisfacción Laboral & $\begin{array}{l}\text { Variable de } 1^{\circ} \text { Orden, } \\
\text { reflectiva }\end{array}$ & 10 & Macdonald y Maclntyre, (1997) \\
\hline Desempeño & $\begin{array}{l}\text { Variable de } 1^{\circ} \text { Orden, } \\
\text { reflectiva }\end{array}$ & 6 & $\begin{array}{l}\text { Ellinger et al., (2008), Babin y } \\
\text { Boles, (1996) }\end{array}$ \\
\hline Conflicto Trabajo-Familia & $\begin{array}{l}\text { Variable de } 1^{\circ} \text { Orden, } \\
\text { reflectiva }\end{array}$ & 5 & Netemeyer et al. (1996) \\
\hline Intención de dejar el trabajo & $\begin{array}{l}\text { Variable de } 1^{\circ} \text { Orden, } \\
\text { reflectiva }\end{array}$ & 3 & Fournier et al., (2010) \\
\hline
\end{tabular}

\section{RESULTADOS Y DISCUSIÓN}

Los criterios de calidad -fiabilidad y confiabilidad- del modelo de mínimos cuadrados parciales (PLS), se presentan en el análisis de consistencia interna y validez convergente, luego en el modelo estructural se verifican las hipótesis planteadas. Primeramente, al evaluar la IE como una variable de segundo orden, con la metodología de dos pasos (Wright et al., 2012), se mantiene la consistencia interna y validez convergente de los constructos (ver tabla 2), se cumple con todos los criterios tanto para las cargas $(\lambda)$, Alpha de Cronbach ( $\alpha$ ) y 
fiabilidad compuesta (FC). Además, las cargas de los cuatro criterios observables del constructo inteligencia emocional, muestran que la autoevaluación emocional y el uso de las emociones tienen una mayor relación con la variabilidad de la inteligencia emocional de los docentes.

La fiabilidad individual de las variables se evaluó a través de las cargas $\lambda$ (Loadings), según afirman Hair et al. (2014), para aceptar un indicador como integrante de un constructo este debe poseer una carga o $\lambda \geq 0,70$ o al menos 0,5 cuando el instrumento se aplica en otros contextos (Chin, 1998), los indicadores cumplen con este criterio. Los resultados para el Alpha de Cronbach ( $\alpha$ ) y fiabilidad compuesta $(F C)$ están por encima del valor recomendado, mayor a 0,7 (Henseler et al., 2016), señalando así que existe consistencia interna en los constructos. Fornell y Larcker (1981), señalan que la fiabilidad compuesta es una medida de consistencia interna superior al alfa de Cronbach, aseverando que es una medida más general que esta última, que además posee la ventaja de no verse influenciada por el número ítems existentes en una escala. Luego, a través de la varianza media extraída (AVE), se calificó la validez convergente de cada constructo, la varianza media extraída (ver tabla 3) está por sobre el nivel recomendado de 0,5 (Chin, 1998). En definitiva, los resultados presentan consistencia interna y una adecuada validez convergente para todos los constructos.

Tabla 2: Criterios para confiabilidad y validez convergente

\begin{tabular}{|c|l|c|c|c|}
\hline \multirow{2}{*}{ Indicador } & Carga $(\lambda)$ & $\begin{array}{c}\alpha \text { de } \\
\text { Cronbach }\end{array}$ & $\begin{array}{c}\text { Fiabilidad } \\
\text { compuesta } \\
\text { (FC) }\end{array}$ \\
\hline \multirow{3}{*}{ IE } & Autoevaluación emocional (AEE) & 0,818 & & \\
\cline { 2 - 3 } & Evaluación emocional de otros (EEO) & 0,687 & \multirow{3}{*}{0,749} & \multirow{2}{*}{0,841} \\
\cline { 2 - 3 } & Regulación de las emociones (RE) & 0,639 & & \\
\cline { 2 - 3 } & Uso de las emociones (AEE) & 0,862 & & \\
\hline
\end{tabular}

Tabla 3: Criterios para evaluación validez convergente del modelo

\begin{tabular}{|c|c|c|c|}
\hline Constructo & $\begin{array}{c}\text { Alfa de } \\
\text { Cronbach } \\
(\alpha)\end{array}$ & $\begin{array}{c}\text { Fiabilidad } \\
\text { compuesta } \\
(F C)\end{array}$ & $\begin{array}{c}\text { Varianza extraída } \\
\text { media (AVE) }\end{array}$ \\
\hline Inteligencia Emocional & 0,749 & 0,841 & 0,573 \\
\hline Satisfacción Laboral & 0,928 & 0,942 & 0,670 \\
\hline Conflicto Trabajo Familia & 0,940 & 0,954 & 0,807 \\
\hline Intención de dejar el trabajo & 0,877 & 0,924 & 0,802 \\
\hline Desempeño & 0,824 & 0,873 & 0,537 \\
\hline
\end{tabular}

Para juzgar la validez discriminante se utilizó el criterio de Fornell-Larcker (Fornell y Larcker, 1981). Según el cual la raíz cuadrada del AVE (en la diagonal principal de la tabla) debe ser mayor a las correlaciones con los otros constructos. Es decir, que en el modelo los constructos comparten más varianza con sus indicadores que con otros constructos. Los resultados de la tabla 4 muestran que todos los constructos cumplen con este criterio.

Tabla 4: Criterio de Forner-Larcker incluida variable de $2^{\circ} \circ$ orden.

\begin{tabular}{|l|c|c|c|c|c|}
\hline & $\begin{array}{c}\text { Conflicto } \\
\text { Trabajo } \\
\text { Familia }\end{array}$ & Desempeño & $\begin{array}{c}\text { Inteligencia } \\
\text { Emocional }\end{array}$ & $\begin{array}{c}\text { Intención de } \\
\text { dejar el } \\
\text { trabajo }\end{array}$ & $\begin{array}{c}\text { Satisfacción } \\
\text { Laboral }\end{array}$ \\
\hline Conflicto Trabajo Familia & 0,898 & & & & \\
\hline Desempeño & $-0,276$ & 0,733 & & & \\
\hline Inteligencia Emocional & $-0,175$ & 0,699 & 0,757 & & \\
\hline Intención de dejar el trabajo & 0,463 & $-0,301$ & $-0,337$ & 0,895 & \\
\hline Satisfacción Laboral & $-0,453$ & 0,524 & 0,504 & $-0,621$ & 0,819 \\
\hline
\end{tabular}

El modelo estructural fue evaluado tanto global como localmente. Respecto a la evaluación global o general, el criterio de ajuste del modelo global es la normalización de raíz cuadrada media residual (SRMR) (Hair et al. 2014). Este criterio considera un ajuste adecuado del modelo cuando los valores son menores a 0,08, en nuestros 
resultados el modelo estimado presenta una SRMR de 0,07. Localmente, el modelo fue evaluado por medio de la significancia estadística de las cargas y estimando los valores de $\mathrm{R}^{2}$. Los valores $\mathrm{p}$ obtenidos a través de boostraping, señalan que todas las relaciones planteadas en el modelo son estadísticamente significativas al $95 \%$ de confianza (ver tabla 5). Los valores de $\mathrm{R}^{2}$, indican la capacidad que tiene un modelo para explicar la variabilidad del constructo. En los resultados el $42,7 \%$ de la varianza de la intención de dejar el trabajo se explica por el conflicto trabajo familia y la satisfacción laboral. A su vez, el 39,1\% de la variabilidad de la satisfacción laboral se explica por el conflicto trabajo familia y por la inteligencia emocional. A sí mismo, más de la mitad de la variación en la percepción del desempeño, se explica por la satisfacción laboral y la inteligencia emocional.

Tabla 5: Test de Hipótesis

\begin{tabular}{|c|l|c|c|c|c|c|}
\hline Hipótesis & \multicolumn{1}{|c|}{ Relación } & $\begin{array}{c}\text { Carga } \\
(\lambda)\end{array}$ & $\begin{array}{l}\text { STDEV } \\
\text { H1 }\end{array}$ & $\begin{array}{c}\text { Estadístico } \\
t\end{array}$ & $\begin{array}{c}\text { Valores } \\
P\end{array}$ & Resultado \\
\hline H2 & Inteligencia Emocional ->Satisfacción Laboral & 0,438 & 0,062 & 7,088 & 0,000 & Aceptada \\
\hline H3 & Inteligencia Emocional ->Desempeño & 0,583 & 0,105 & 5,565 & 0,000 & Aceptada \\
\hline H4 & Conflicto Trabajo Familia ->Satisfacción Laboral & $-0,376$ & 0,083 & 4,547 & 0,000 & Aceptada \\
\hline H5 & $\begin{array}{l}\text { Conflicto Trabajo Familia ->Intención de dejar el } \\
\text { trabajo }\end{array}$ & 0,228 & 0,094 & 2,410 & 0,016 & Aceptada \\
\hline H6 & Satisfacción Laboral ->Desempeño & 0,230 & 0,097 & 2,372 & 0,018 & Aceptada \\
\hline H7 & Satisfacción Laboral ->Intención de dejar el trabajo & $-0,518$ & 0,083 & 6,220 & 0,000 & Aceptada \\
\hline
\end{tabular}

Adicionalmente, se evaluó la relevancia predictiva del modelo estructural, a través del test Stone-Geisser Q2 (Geisser, 1974). Al respecto, Chin (1998) menciona que la relevancia predictiva de los constructos debe ser positiva y con valores mayores a cero; así también establecen valores de 0,02 como valores pequeños, valores de 0,15 como valores medios y valores de 0,35 como valores grandes para considerar validez predictiva del modelo. Según los resultados (ver figura 2), el modelo posee una relevancia predictiva entre pequeña y media. El único constructo para el cual el modelo tiene una pequeña relevancia predictiva es el conflicto trabajo familia. De lo que se infiere que la mayor parte de la variabilidad en el CTF se explica por factores que no están incluidos en el modelo.

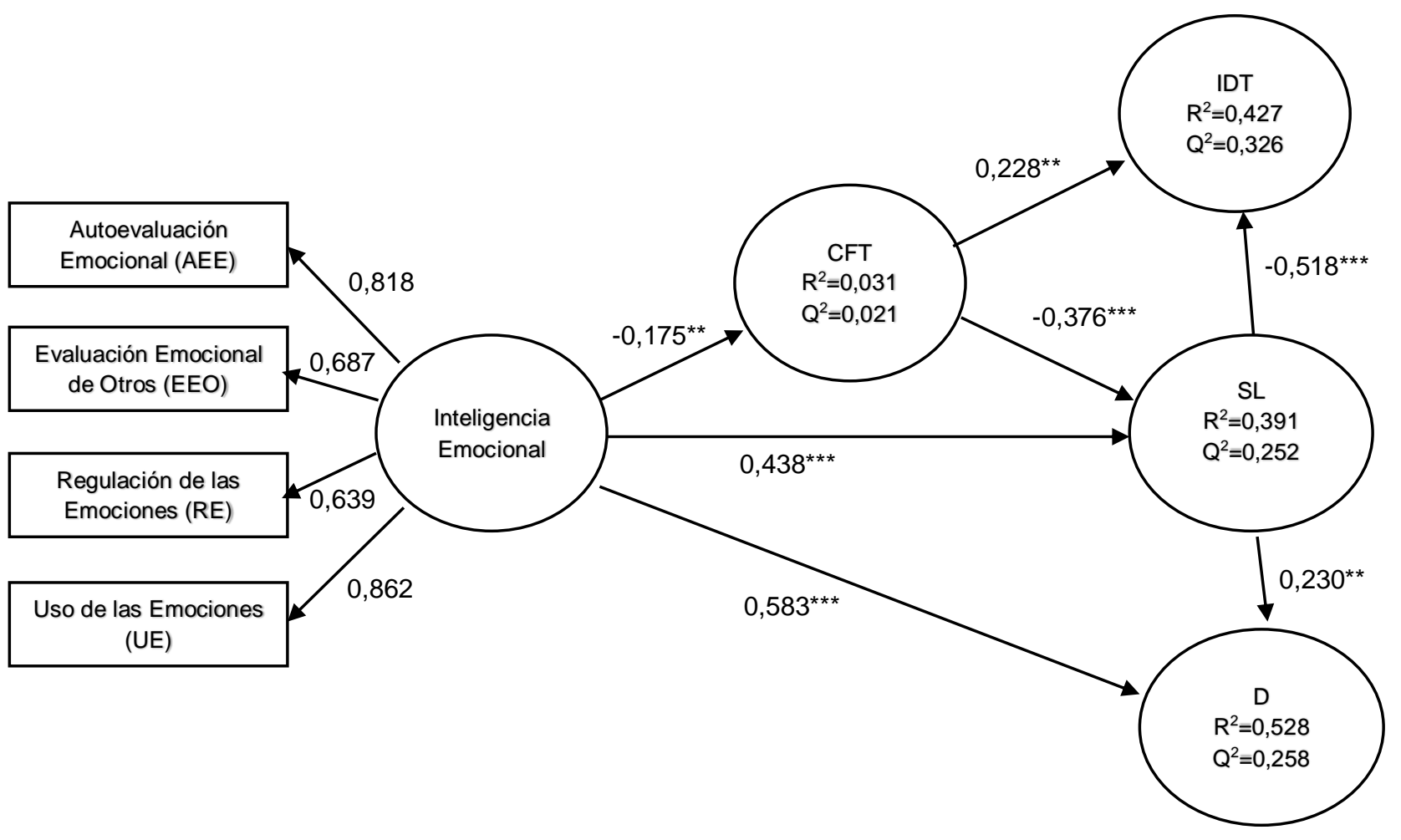

Figura 2: Resultados 
Al igual que en el trabajo de Sy et al. (2006), los resultados muestran una relación positiva y directa entre la inteligencia emocional, la satisfacción laboral y el desempeño (Ver figura 2). Por otra parte, y en línea con lo planteado por Gao et al. (2013), la inteligencia emocional se relación de forma inversa con el conflicto trabajofamilia, facilitando la regulación del conflicto. A su vez, los resultados muestran que el CTF aumenta la intención de dejar el trabajo (IDT), tanto en forma directa, como indirecta, al igual que en el trabajo de Erdamar y Demirel (2016). Además, el CTF disminuye la satisfacción laboral, puesto que presentan una relación directa y negativa, manteniendo la relación obtenida por el trabajo de Johnsrud y Rosser (2002). Destaca en el modelo estructural la importante y significativa carga de la satisfacción laboral en la intención de abandono del trabajo. En definitiva, el estudio confirma que la ocupación docente mantiene las mismas relaciones de causalidad de las ocupaciones gerenciales o directivas (presentadas en las hipótesis).

Los docentes desempeñan un rol de primera línea en el relacionamiento con la comunidad educativa, en especial con los alumnos. Esto los vuelve determinantes en el logro de resultados dentro de los establecimientos educacionales, sin embargo, estos están sujetos a un importante desgaste emocional (Wen et al., 2019). En donde, la inteligencia emocional y las estrategias que utilizan para realizar el trabajo emocional son extremadamente importantes para el éxito de su enseñanza, así como para su bienestar psicológico (Yin et al., 2013). En este trabajo confirmamos que la IE tiene un papel preponderante en el desempeño y la satisfacción laboral de los docentes. Con especial relevancia, destacan las dimensiones de autoevaluación emocional y el uso de las emociones. Elementos que se deberían de considerar en los programas de desarrollo de competencias en inteligencia emocional para docentes.

Respecto al CTF, se verifica que la inteligencia emocional puede tener una relación significativa en la variabilidad de este factor, pero con un bajo impacto y poca capacidad explicativa. En la medición del CTF, los docentes manifiestan que las demandas labores pueden llegar a sobrepasar su jornada laboral, interrumpiendo su tiempo familiar. Aquí es importante mencionar que la muestra es mayoritariamente femenina (81\%), lo que podría exacerbar esta medición. Puesto que, distintos estudios señalan que las mujeres docentes experimentan más conflictos entre el trabajo y la familia que los hombres (Erdamar y Demirel, 2014). Además, en este estudio se demuestra que a medida que aumenta el CTF aumenta la intención de abandono del trabajo y disminuye la satisfacción laboral. Esto vuelve especialmente relevante el desarrollo de políticas organizacionales en establecimientos educacionales que permitan compatibilizar el rol docente y familiar.

La satisfacción laboral del docente se relaciona significativamente con la intención de abandono y el desempeño percibido del docente. Debido a que, en la medida que disminuye la satisfacción laboral, se pueden presentar aspectos negativos del comportamiento organizacional; tales como el ausentismo, la rotación en el trabajo, y la baja productividad. Lo que, a su vez, puede afectar el bienestar de los docentes y los resultados de los establecimientos educacionales.

\section{CONCLUSIONES}

De acuerdo con el trabajo presentado y a los resultados obtenidos, se pueden plantear las siguientes conclusiones principales:

1.- En el contexto de docentes de primaria -principalmente mujeres- de establecimientos particulares subvencionados en Chile, se verifican las hipótesis planteadas; es decir que la inteligencia emocional se relaciona directa y positivamente con la satisfacción laboral y el desempeño percibido, y negativamente con el conflicto trabajo familia.

2.- El conflicto trabajo familia tiene un efecto directo negativo en la satisfacción laboral y un efecto directo positivo en la intención de dejar el trabajo

3.- La satisfacción laboral tiene un importante efecto directo negativo sobre la intención de dejar el trabajo, y un efecto directo positivo sobre el desempeño.

\section{REFERENCIAS}

Babin, B. J y Boles, J. S., The effects of perceived co-worker involvement and supervisor support on service provider role stress, performance and job satisfaction, https://doi.org/10.1016/S0022-4359(96)90005-6, Journal of Retailing, 72(1), 5775 (1996).

Bar-on, R., Bar-On Emotional Quotient Inventory (EQ-i): Technical manual. Toronto: Multi-Health Systems. In Bar-On, R. y Parker, D. (eds.), The handbook of emotional intelligence. San Francisco: Jossey-Bass (1997).

Bustamante, M. A., Álvarez, A. J., Villalobos, M. E., y Lucero, M. I., Percepción de la calidad de vida laboral de los trabajadores de los centros de salud familiar de la zona central de Chile, http://dx.doi.org/10.4067/S071807642020000300065, Información tecnológica, 31(3), 65-74 (2020). 
Cheung, F. Y., y Tang, C. S., The Effect of Emotional Dissonance and Emotional Intelligence on Work-Family Interference, https://doi.org/10.1037/a0025798, Canadian Journal of Behavioral Science, 44(1), 50-58 (2012).

Chin, W. W., The partial least squares approach to structural equation modeling, Modern methods for business research, 295(2), 295-336 (1998).

Ellinger, A. E., Ketchen, D. J., y otros tres autores., Market orientation, employee development practices, and performance in logistics service provider firms, https://doi.org/10.1016/j.indmarman.2007.01.002,_Industrial Marketing Management, 37(4), 353-366 (2008).

Erdamar, G. y Demirel, H., Investigation of Work-family, Family-work Conflict of the Teachers, https://doi.org/10.1016/j.sbspro.2014.01.1050, Procedia - Social and Behavioral Sciences, 116, 4919-4924 (2014).

Erdamar, G. y Demirel, H., Job and Life Satisfaction of Teachers and the Conflicts They Experience at Work and at Home, https://doi.org/10.11114/jets.v4i6.1502, Journal of Education and Training Studies, 4, 164-175 (2016).

Fournier, C., Tanner, J. F., Chonko, L. B. y Manolis, C., The Moderating Role of Ethical Climate on Salesperson Propensity to Leave, http://dx.doi.org/10.2753/PSS0885-3134300101, Journal of Personal Selling \& Sales Management, 30(1), 7-22 (2010).

Fornell, C. y Larcker, D. F., Evaluating structural equation models with unobservable variables and measurement error, https://doi.org/10.2307/3151312, Journal of marketing research, 18(1), $39-50$ (1981).

Gao, Y., Shi, J., Niu, Q. y Wang, L., Work-Family Conflict and Job Satisfaction: Emotional Intelligence as a Moderator. https://doi.org/10.1002/smi.2451. Stress and Health, 29, 222-228 (2013).

Geisser, S., A predictive approach to the random effect model, https://doi.org/10.2307/2334290, Biometrika, 61(1), 101 107 (1974).

Greenhaus, J. H., y Beutell, N. J., Sources of conflict between work and family roles. https://doi.org/10.5465/amr.1985.4277352 Academy of Management Review, 10(1), 76-88 (1985).

Gudergan, S. P., Ringle, C. M., Wende, S., y Will, A., Confirmatory tetrad analysis in PLS path modeling, https://doi.org/10.1016/j.jbusres.2008.01.012, Journal of business research, 61(12), 1238-1249 (2008).

Hair Jr, J., Sarstedt, M., Hopkins, L. y Kuppelwieser, V.G., Partial least squares structural equation modeling (PLS-SEM) An emerging tool in business research, https://doi.org/10.1108/EBR-10-2013-0128, European Business Review, 26(2), 106-121 (2014).

Henseler, J., Hubona, G. y Ray, P. A., Using PLS path modeling in new technology research: updated guidelines, https://doi.org/10.1108/IMDS-09-2015-0382, Industrial management y data systems, 116(1), 2-20 (2016).

Johnsrud, L. K. y Vicki J. R., Faculty Members' Morale and their Intentions to Leave. A Multilevel Explanation, The Journal of Higher Education, 73(4), 518-542 (2002).

Joseph, D. L., Jin, J., Newman, D. A., y O'Boyle, E. H., Why does self-reported emotional intelligence predict job performance? A meta-analytic investigation of mixed El, https://doi.org/10.1037/a0037681, Journal of Applied Psychology, 100(2), 298-342 (2015).

Macdonald, S. y Maclntyre, P., The Generic Job Satisfaction Scale, https://doi.org/10.1300/J022v13n02_01, Employee Assistance Quarterly, 13(2), 1-16 (1997).

Mayer, J. D., y Salovey, P., The intelligence of emotional intelligence, https://doi.org/10.1016/0160-2896(93)90010-3, Intelligence, 17(4), 433-442 (1993).

Netemeyer, R. G., Boles, J. S., y McMurrian, R., Development and validation of work-family conflict and family-work conflict scales, https://doi.org/10.1037/0021-9010.81.4.400, Journal of Applied Psychology, 81(4), 400-410 (1996).

Patterson, M., Warr, P., y West, M., Organizational climate and company productivity: the role of employee affect and employee level, https://doi.org/10.1348/096317904774202144, Journal of Occupational and Organizational Psychology, 77, 193-216 (2004).

Petrides, K. V., Pita, R., y Kokkinaki, F., The location of trait emotional intelligence in personality factor space, https://doi.org/10.1348/000712606x120618, British journal of psychology, 98(2), 273-289 (2007).

Porter L. M. y Lawler E. E., Actitud empresarial y desempeño. New York, McGraw-Hill, (1991).

Pousette, A., y Hanse, J. J., Job characteristics as predictors of ill-health and sickness absenteeism in different occupational types--a multigroup structural equation modelling approach, https://doi.org/10.1080/02678370210162737, Work and Stress, 16, 229-250 (2002).

Ringle, C. M., Wende, S., y Becker, J. M., SmartPLS 3. SmartPLS GmbH, Boenningstedt. (2015).

Shi, J., y Wang, L., Validation of emotional intelligence scale in Chinese university students, https://doi.org/10.1016/j.paid.2006.12.012, Personality and Individual Differences, 43(2), 377-387 (2007).

Sy, T., Tram, S., y O'Hara, L. A., Relation of employee and manager emotional intelligence to job satisfaction and performance, https://doi.org/10.1016/j.jvb.2005.10.003, Journal of vocational behavior, 68(3), 461-473 (2006). 
Wen, J., Huang, S. S., y Hou, P., Emotional intelligence, emotional labor, perceived organizational support, and job satisfaction: A moderated mediation model, https://doi.org/10.1016/j.ijhm.2019.01.009, International Journal of Hospitality Management, 81, 120-130 (2019).

Wong, C. S., y Law, K. S., The effects of leader and follower emotional intelligence on performance and attitude: An exploratory study, https://doi.org/10.1016/S1048-9843(02)00099-1, The leadership quarterly, 13(3), 243-274 (2002).

Wong, C. S., Wong, P. M., y Peng, K. Z., Effect of middle-level leader and teacher emotional intelligence on school teachers' job satisfaction: The case of Hong Kong, https://doi.org/10.1177\%2F1741143209351831, Educational Management Administration \& Leadership, 38(1), 59-70 (2010).

Wright, R. T., Campbell, D. E., Thatcher, J. B. y Roberts, N., Operationalizing multidimensional constructs in structural equation modeling: recommendations for is research, https://doi.org/10.17705/1CAIS.03023, Communications of the Association for Information Systems, 30(23), 367-412 (2012).

Yin, H. B., Lee, J. C. K., y Zhang, Z. H., Exploring the relationship among teachers' emotional intelligence, emotional labor strategies and teaching satisfaction, https://doi.org/10.1016/j.tate.2013.06.006, Teaching and teacher education, 35, 137145 (2013).

Yoke, L. B., y Panatik, S. A., Emotional intelligence and job performance among school teachers, https://doi.org/10.5539/ass.v11n13p227, Asian Social Science, 11(13), 227-234 (2015). 
